\title{
Bioinformatics Analysis of the Gene CYP83B1 in Cabbage (Brassica oleracea var. capitata)
}

\author{
Bo Sun, Min Jiang, Qiao Yuan, Fen Zhang, and Haoru Tang* \\ College of Horticulture, Sichuan Agricultural University, Chengdu 611130, China. \\ *Correspondence author, E-mail addresses: htang@sicau.edu.cn.
}

Keywords: Cabbage, carotenoids, CYP83B1, bioinformatics analysis.

\begin{abstract}
CYP83B1 is an important glucosyltransferase enzyme in glucosinolate biosynthesis. Here, the Brassica oleracea var. capitata CYP83B1 (BocCYP83B1) gene sequence was obtained from Brassica database (BRAD), and preformed for bioinformatics analysis. The BocCYP83B1 gene mapped to chromosomes 8 , and contains an open reading frame of 1,473 bp that encodes a 490 -amino acid protein with a calculated molecular mass of $55.89 \mathrm{kD}$ and an isoelectric point (pI) of 8.89. Subcellular localization predicted the BocCYP83B1 gene was in the chloroplast. The conserved domain of the BocCYP83B1 protein is belonged the p450 superfamily. The CYP83B1 protein is most closely related to $B$. rapa. The findings of the present study provide a molecular basis for the elucidation of CYP83B1 gene function in cabbage.
\end{abstract}

\section{Introduction}

Cabbage (Brassica oleracea var. capitata) is a member of the Brassicaceae family that is widely distributed in the world. In China, cabbage is an important vegetable crop, and consumed considerable every year. Cabbage is generally grown for its leafy head as common edible part, which are crispy, tender, and tasty [1]. Besides its good flavor, cabbage is also a rich source of nutrients, antioxidants, and anticarcinogenic compounds, including carbohydrates, vitamin C, carotenoids, and glucosinolates [1-2].

Glucosinolates are a group of sulfur- and nitrogen-containing secondary metabolites that are mainly found in the order of Brassicales and related groups of dicotyledonous angiosperms [3-4]. Glucosinolates and the hydrolytic myrosinase ( $\beta$-thiosglucoside glucohydrolase) are stored separately under normal situations, but they come into contact with each other when tissues are damaged, and then the glucosinolates are hydrolyzed into several degradation products, such as isothiocyanates and nitriles [5]. Glucosinolates and their degradation products have diverse biological functions, which contribute to human health, as well as the taste and odor of cruciferous crops. The anticancer activity of isothiocyanates has been widely studied, and the mechanism involved has been elucidated [6].

Glucosinolate metabolism in plants is modulated by numerous biotic and abiotic factors, and the regulatory network of glucosinolate metabolism has been well elucidated in Arabidopsis [6]. CYP83B1 is metabolized for aromatic oximes, and has higher affinity, particularly in the case of indole-3-acetaldoxime [7]. The gene encoding the CYP83B1 protein has been isolated in Arabidopsis thaliana and Chinese cabbage [7]. To date, research studies on CYP83B1 in cabbage are limited. In the present study, the CYP83B1 gene sequence of cabbage was obtained from web database, and then bioinformatics analysis of the CYP83B1 gene were analyzed. The present study aimed to establish the foundation for further studies on the molecular mechanism of CYP83B1 in cabbage. 


\section{Materials and methods}

\subsection{Sequence Obtain of the BocCYP83B1 Gene.}

The genomic DNA and mRNA sequences of CYP83B1 gene of cabbage were downloaded and obtained from The Brassica database (BRAD) (http://brassicadb.org), and then used to subsequent bioinformatic analysis.

\subsection{Bioinformatics Analysis of the BocCYP83B1 Gene.}

The amino acid sequence, protein molecular weight, isoelectric point, stability index, and hydrophobicity of the BocCYP83B1 gene were analyzed and predicted by ExPASy (http://web.expasy.org) and NCBI (https://www.ncbi.nlm.nih.gov/). Subcellular localization was predicted by WoLF PSORT (http://www.genscript.com/wolf-psort.html). The conserved domain were predicted by NCBI (https://www.ncbi.nlm.nih.gov/Structure/cdd/wrpsb.cgi). Phylogenetic tree analysis of the CYP83B1 proteins was executed in MEGA 6.0 using the neighbor-joining (NJ) method.

\section{Results}

\subsection{Analysis on Genomic Organization.}

The Brassica database (BRAD) was used to analyze the chromosomal localization and genomic organization of BocCYP83B1. The gene ID in BRAD is Bol033477. The BocCYP83B1 gene was mapped to chromosomes 8 and has 2 exons and 1 intron (Fig. 1).

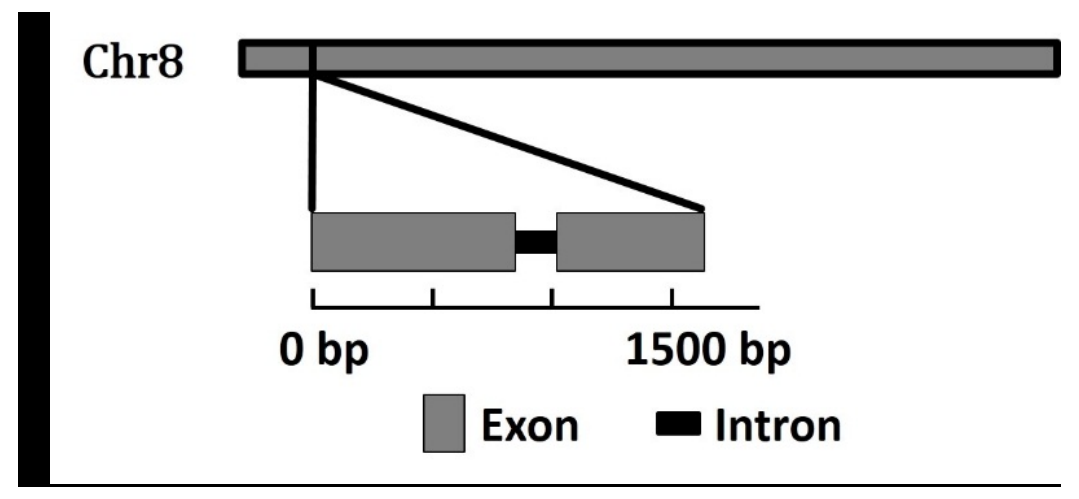

Fig. 1 Chromosomal location and genomic structure of BocCYP83B1.

\subsection{Protein Physical and Chemical Properties Analysis.}

Sequence analysis indicated that the BocCYP83B1 gene contained a 1,473-bp open reading frame (ORF), which encoded a 490-amino acids protein with a calculated molecular mass of $55.89 \mathrm{kD}$ and an isoelectric point (pI) of 8.89. The amino acid types and proportions of the BocCYP83B1 gene was shown in Figure 2, the highest number of amino acid is Leucine (Leu), whereas the lowest number is Tryptophan (Trp). Its predicted formula was $\mathrm{C}_{2538} \mathrm{H}_{3994} \mathrm{~N}_{664} \mathrm{O}_{703} \mathrm{~S}_{26}$. Its total average hydrophilicity index was -0.158 , liposoluble index was 89.78 , and instability index in solution was 36.88. 


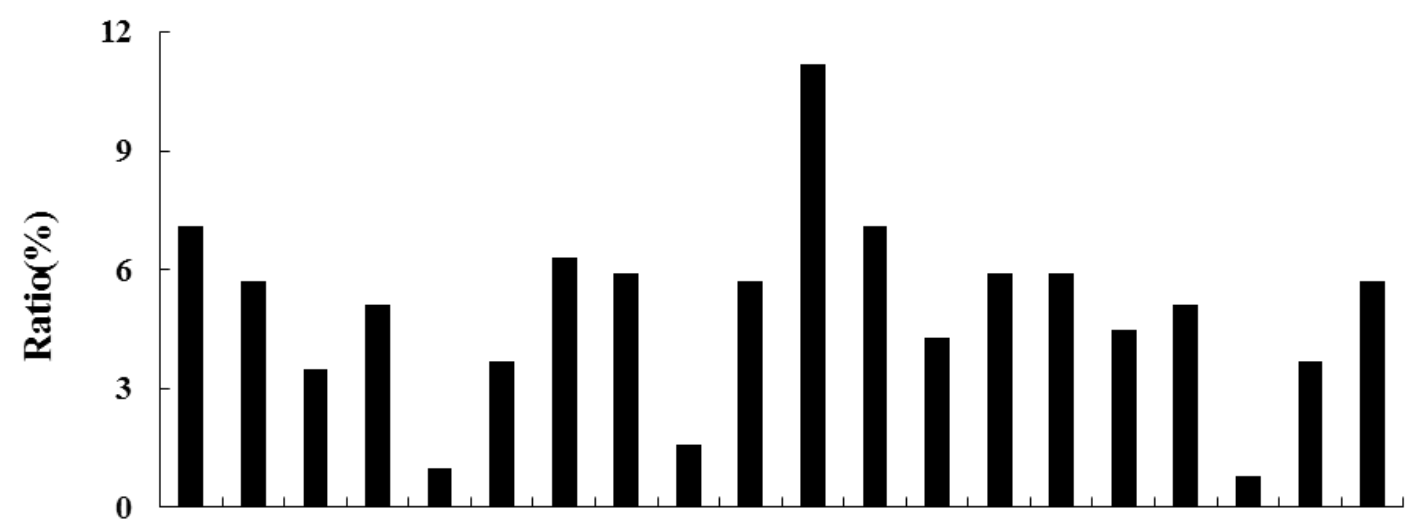

Ala Arg Asn Asp Cys Gln Glu Gly His Ile Leu Lys Met Phe Pro Ser Thr Trp Tyr Val

Amino Acid

Fig. 2 Amino acid composition of BocCYP83B1

\subsection{Subcellular Localization and Conserved Domain Analysis.}

Subcellular localization of the BocCYP83B1 gene was predicted by WoLF PSORT to be in the chloroplast. The analysis using Conserved Domain Database (CDD) demonstrated that the amino acid sequence of the BocCYP83B1 protein belonged the p450 superfamily.

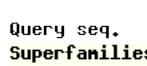

p450 superfamily

Fig. 3 Conserved domains analysis of BocCYP83B1

\subsection{Homology and Phylogenetic Tree Analysis.}

A phylogenetic tree was constructed to illustrate the relationship among the CYP83B1 proteins of cabbage and 18 other higher plant species (Fig. 4). A total of two major clusters were identified. Sequence alignment indicated that the BocCYP83B1 protein is more closely related to B. rapa.

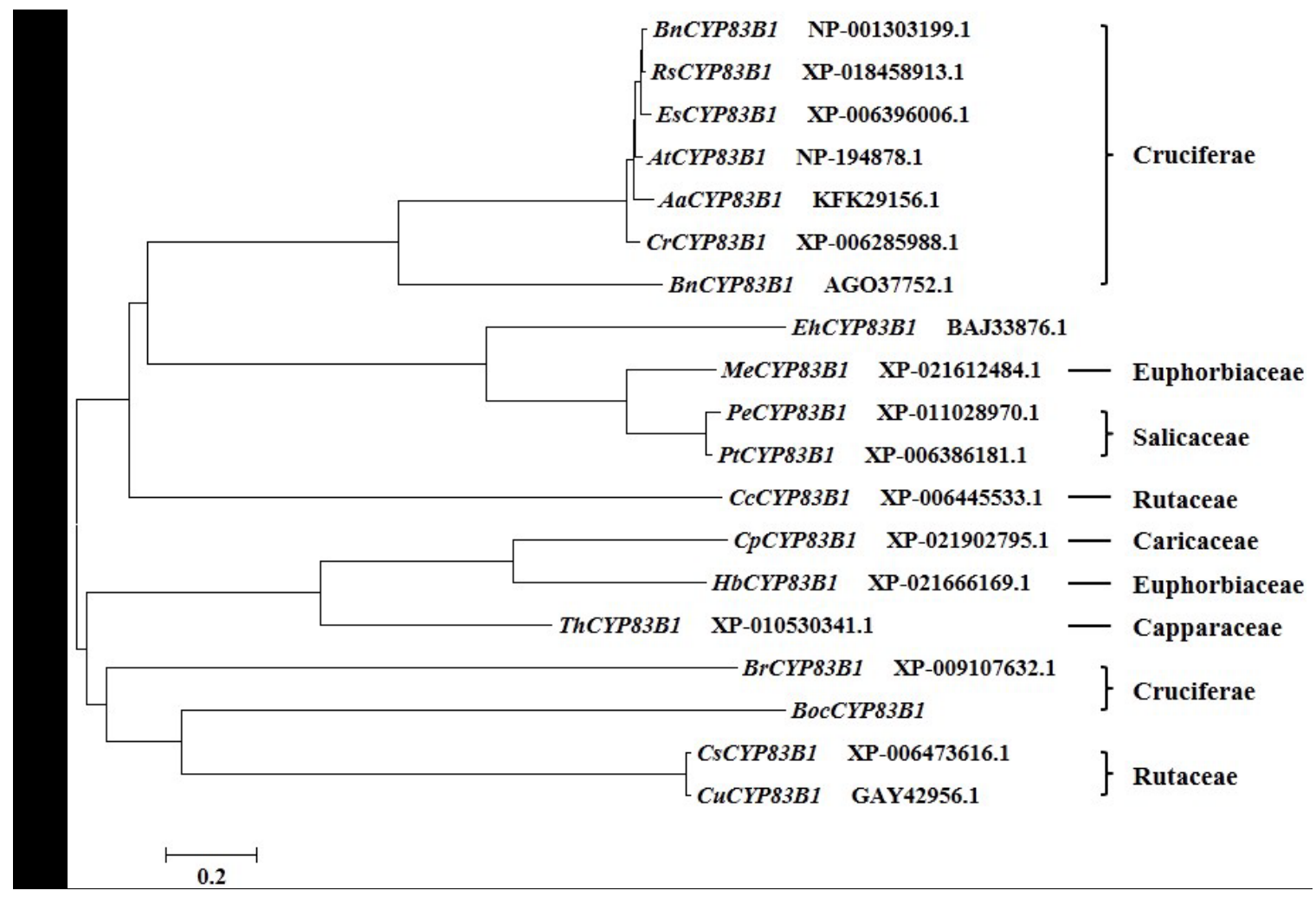

Fig. 4 Phylogenetic tree analysis of BocCYP83B1 and CYP83B1 proteins of other species 


\section{Discussion}

The present study analyzed the BocCYP83B1 gene of cabbage. CYP83B1 enzyme is encoded by a single-copy gene in Arabidopsis thaliana [7]. While, the CYP83B1 gene occurred as a single copy in cabbage, indicating that the enzyme may have undergone similar evolutionary patterns. Previous studies have shown that the CYP83B1 protein is relatively conserved in plants [7]. The findings of the present study show that CYP83B1 from cabbage is highly conserved in plants, similar to that observed in earlier reports. The findings of the present study may serve as a foundation for future studies on the functions of CYP83B1 in glucosinolate metabolism in cabbage.

\section{Acknowledgements}

This work was supported by National Natural Science Foundation of China (31500247), key project of Department of Education of Sichuan Province (14ZA0016), and National Student Innovation Training Program (201710626030).

\section{References}

[1] M. Wennberg, J. Ekvall, K. Olsson, and M. Nyman, Changes in carbohydrate and glucosinolate composition in white cabbage (Brassica oleracea var. capitata) during blanching and treatment with acetic acid, Food Chem. 95 (2006) 226-236.

[2] S. Rokayya, C.J. Li, Y. Zhao, Y. Li, and C.H. Sun, Cabbage (Brassica oleracea L. var. capitata) phytochemicals with antioxidant and anti-inflammatory potential, Asian Pac. J. Cancer Prev. 14 (2014) 6657-6662.

[3] B.G. Hansen, R.E. Kerwin, J.A. Ober, V.M. Lambrix, T. Mitchell-Olds, J. Gershenzon, B.A. Halkier, and D.J. Kliebenstein, A novel 2-oxoacid-dependent dioxygenase involved in the formation of the goiterogenic 2-hydroxybut-3-enyl glucosinolate and generalist insect resistance in Arabidopsis, Plant Physiol. 148 (2008) 2096-2108.

[4] X. Yan, and S. Chen. Regulation of plant glucosinolate metabolism, Planta 226 (2007) 1343-1352.

[5] E. Andréasson, L.B. Jørgensen, A.S. Höglund, L. Rask, and J. Meijer, Different myrosinase and idioblast distribution in Arabidopsis and Brassica napus, Plant Physiol. 127 (2001) 1750-1763.

[6] N. Benkeblia, Phytonutritional Improvement of Crops, John Wiley \& Sons, Inc., Hoboken, 2017, pp. 407-733.

[7] S. Bak, F.E. Tax, K.A. Feldmann, D.W. Galbraith, and R. Feyereisen, CYP83B1, a cytochrome $\mathrm{P} 450$ at the metabolic branch point in auxin and indole glucosinolate biosynthesis in Arabidopsis, Plant Cell 13 (2001) 101-111. 